\title{
therapeutic approach
}

Isaac Fernández ${ }^{1 \&}$, Marquiza Sablón-Carranzana1,2, \& Alberto Bencomo ${ }^{1,2}$, Reyna Lara-Martínez ${ }^{3}$, Guadalupe Domínguez ${ }^{4}$ Karina Pasten-Hidalgo ${ }^{5,6}$, Rosa Angélica Castillo-Rodríguez ${ }^{5,6}$, Nelly F. Altamirano-Bustamante ${ }^{5}$, Luis Felipe Jiménez-García ${ }^{3}$, Eulalia Garrido-Magaña $^{7}$, Sergio Islas-Andrade ${ }^{1}$, Chryslaine Rodríguez-Tanty ${ }^{*}$ and Myriam M. Altamirano Bustamante ${ }^{1^{*}}$.

'Unidad de Investigación Médica en Enfermedades Metabólicas, Hospital de Cardiología, Centro Médico Nacional Siglo XXI, Instituto Mexicano del Seguro Social. México D.F.

pto. Neurodiagnostico, Centro de Neurociencias de Cuba Cubanacan, Playa, La Habana, Cuba.

Laboratorio de Nanobiología Celular, Departamento de Biología Celular. Facultad de Ciencias, UNAM. México, D. F.

Instituto de Fisiologia Celular, UNAM. México, D. F.

Servicio de Endocrinología, Instituto Nacional de Pediatría, SS México, D. F.

Servicio de Endocrinología, Hospital de Pediatría, CMNXXI, IMSS. México, D. F.

Conch MSC contibuted equally to this work.

\section{OBJECTIVES}

\section{METHODS}

Diabetes mellitus type 2 (T2DM) is correlated with functional tissue loss due to accumulation and aggregation of small peptides, such as the human islet amyloid polypeptide (hIAPP) in the pancreas ${ }^{1,3,4}$. The process of amyloid formation involves aggregation of monomers to form cytotoxic oligomers and then fibrils ${ }^{5}$.

Our objective was to investigate the potential effect of chemical chaperones ${ }^{2}$ in preventing hIAPP aggregation and avoid cytotoxicity. We synthesized a novel family of chaperones derived from naphthalene $(A, B, C, D, E, F$ and $G)^{7,8}$ and analyzed the interaction zones between the hIAPP amino acids. Then, we have carried out an in vitro fluorescencebased fibrillation study. We have also evaluated the effect of chaperones to reduce the cytotoxicity of hIAPP oligomers in cells.
Preparation and characterization of chaperones. Chaperones A, B, C, D, E, F and G were synthesized and purified by Dr. Marquiza at Cuba Neuroscience Center (Havana, Cuba) ${ }^{6,7}$

Oligomer preparation. hIAPP oligomers were prepared essentially as described ${ }^{5}$.

Molecular docking. The crystal structure of IAPP (PDB entry 2L86) was obtained from the Protein Data Bank. The structures of the chemical chaperones were refined using Avogadro program and converts it into pdbqt format with the program Autodock Tools.

Thioflavin $T$ fluorescence assay. hIAPP fibril formation in the presence or absence of chaperones $A, B, C, D, E, F$ and $G$ was monitored using thioflavin $T$ (ThT) fluorescence. Fluorescence measurements were taken using M1000 (Tecan, Austria).

Cell Cultures. Cerebellar granule neurons (CGN) were routinely grown in a $5 \% \mathrm{CO} 2$ humidified incubator at $37^{\circ} \mathrm{C}$ in a 1:1 mixture of MEN medium supplemented with $25 \mathrm{mM}$ $(\mathrm{KCl})$

MTT assay. Reduction of MTT (3-[4,5-dimethylthiazol-2-yl]-2,5-diphenyl tetrazolium bromide) was used to assess cell viability as described previously 4 .

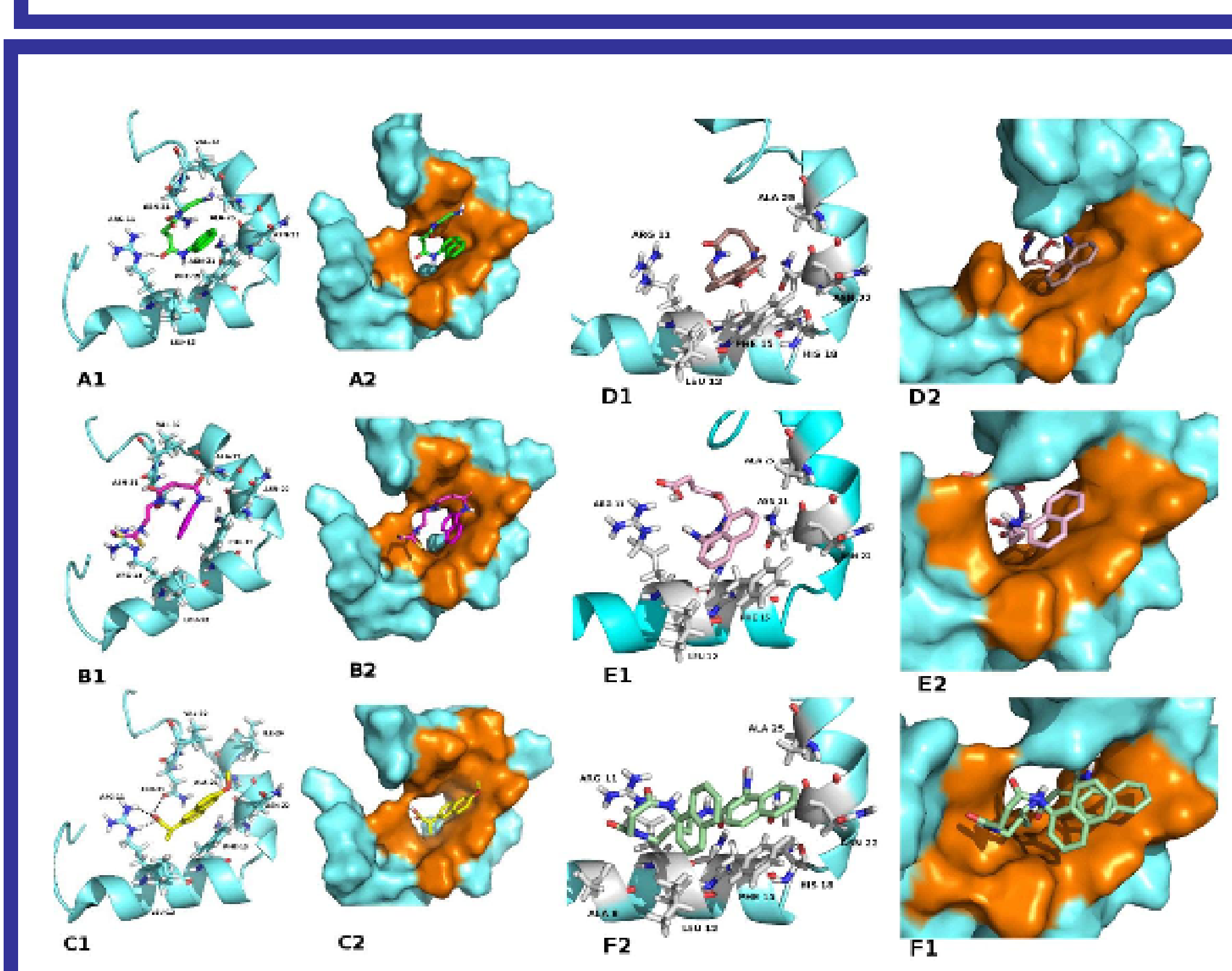

Fig 1. Molecular docked model of most probable interaction of chemical chaperones with hIAPP. At $5 \AA$ distance, the amino acids residues surrounding chap.
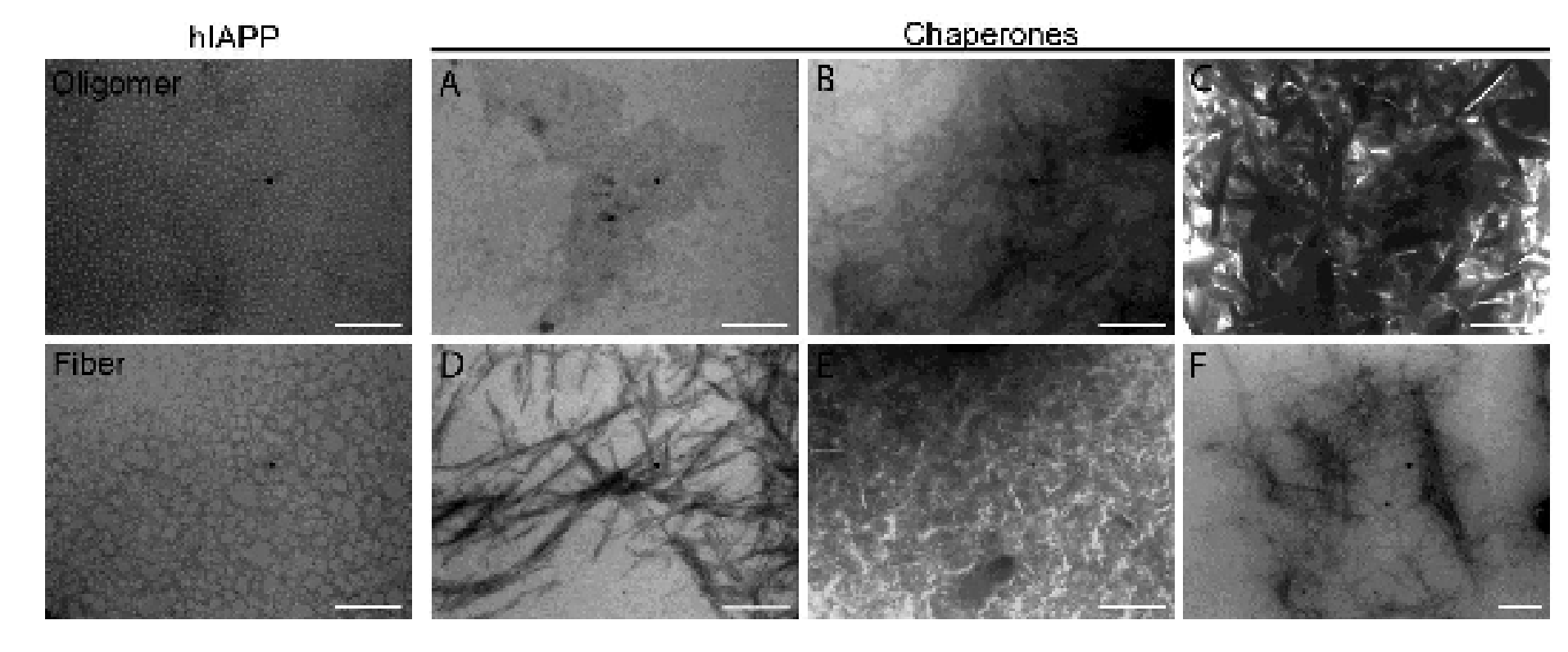

Fig 3. Morphological analysis of hIAPP fibrils with an without chaperones (A, B, C, D, E and F) by TEM. Scal
bar are $200 \mathrm{~nm}$.

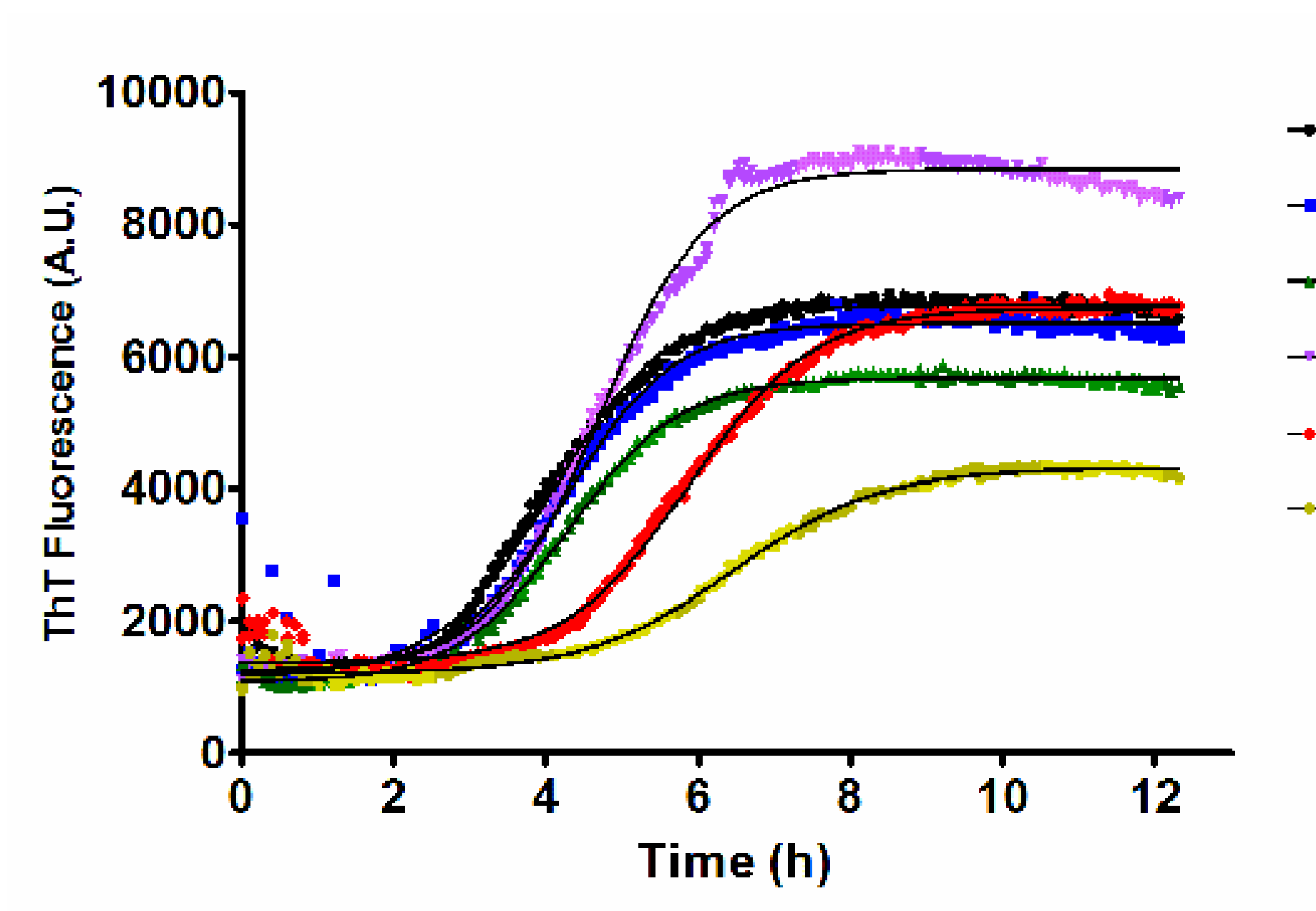

Fig. 2. Kinetics of aggregation of hIAPP and the mixture of hIAPP with chaperones $A, B, C, D$ and $E$ monitored by ThT
fluorescence assays. The concentration of hIAPP was $12.8 \mu \mathrm{M}$ fluorescence assays. The concentration of hIAPP was $12.8 \mu \mathrm{M}$ and $6.4 \mu \mathrm{M}$ for the chaperones. The experiments wer

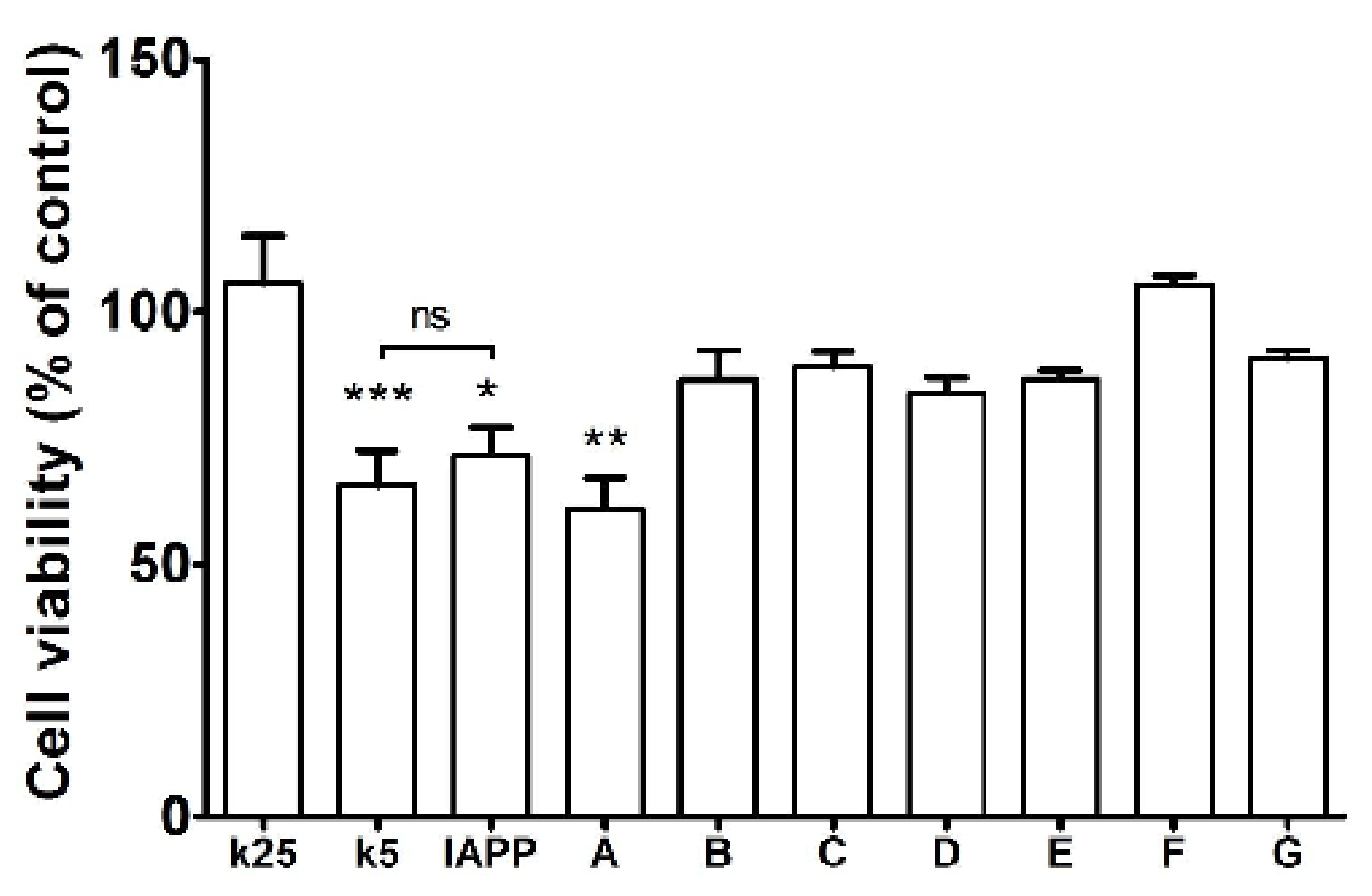

Fig 4. Cytoprotection of the chaperones from the cytotoxic effects of hIAPP aggregation. CGN cells were treated with $80 \mu \mathrm{M}$ of hlAPP
lus $40 \mu \mathrm{M}$ of each chaperone. All conditions, except $\mathrm{K} 5$, were incubated with DMSO $0.8 \%$. K 25 and K5 are potassium $25 \mathrm{mM}$ an

\section{RESULTS}

In the docking model, all the chaperones interact in the same pseudocavity formed in the helical IAPP (Fig.1). It is generated by the interaction between the $\mathrm{H}$ atom of the Arg11 and the $\mathrm{O}$ atom of the Asn31, at a distance of $3.7 \AA$. Overall, the bridging $\mathrm{H}$ takes place from a distance of 3 $\AA$, between the involved atoms. Therefore, we could describe this interaction as a quasi $\mathrm{H}$ bridge

We observed a sigmoidal curve describing the increase in the density of hIAPP fiber formation reveled as Thioflavin (Tht) fluorescence over the time (Fig. 2). Only the chaperone $C$ accelerates and increase the number of fiber formation, as the chaperones $B, D, E$ and, in less proportion chaperone $A$, delays the fiber formation

We observed that the presence of chaperones at the end of the experiment increase the fibril formation compared with the normal aggregation of the IAPP as control. This corroborates the results from the Tht assay (Fig. 3). In some cases as Chaperone $C$ at equimolar doses, we observed even a shape that suggest crystallization

To test cell viability, we incubated the granular cells from mouse cerebellum (CGN) with hIAPP and hIAPP plus each chaperone. Except $A$, the chaperones increase the viability of cells as we observed in the MTT assays (Fig. 4).

\section{CONCLUSIONS}

All the chaperones interact in the same pseudo-cavity formed in the helical IAPP.

According to the kinetics assays, only the chaperone $C$ accelerates and increase the number of fiber formation, as the chaperones $B, D, E$ and, in less proportion chaperone $A$, delays the fiber formation. We think that the chaperones can lead to a stabilization from cytotoxic oligomers to fibers avoiding the toxic effect, but also producing a regression from cytotoxic oligomers to monomers depending of the dose of the chaperone.

Except chaperone $A$, the chaperones rescue the cells from the cytotoxic effect of the hIAPP oligomers, and then could be probed as therapeutic approach in the future.

\section{References} disease and type 2 diabetes mellitus. CNS Neurol Disord Drud Gao M, et. al. (2015) Modulation of human IAPP fibrillation: cosolutes crowders and chaperones. Phys Chem Chem Phys 17(13):8338-8348. Fernandez MS (2014) Human IAPP amyloidogenic properties and pancreatic -cell death. Cell Calcium. 56(5):416-427. 作 type-2 diabetes mellitus. Nature.368:756-760. Kaved R and Glabe CG (2006) Conformation-dependent anti-amyloid oligomer antibodies. Methods Enzymol. 413:326-344 Sablón C. M et. al. Procedimiento de obtención de nuevos derivados de naftaleno para el diagnóstico in vivo de la enfermedad de Alzheimer. ittp://www.google.stpatents/W010118706A2?cl=cle molecular modulators of beta protein aggregation present in conformational 